\title{
Morfologia macroscópica do aparelho reprodutor feminino de Leontopithecus cativos (Lesson, 1840) Primates-Callitrichidae
}

[Gross morphology of the female genital tract of captive Leontopithecus (Lesson, 1840) Primates-Callitrichidae]

\author{
L. Pissinatti ${ }^{1}$, R. Tortelly ${ }^{2}$, M. Porto ${ }^{3}$, C.H.F. Burity ${ }^{4}$, A. Pissinatti ${ }^{3,5}$ \\ ${ }^{1}$ Aluno de pós-graduação - UFF - Niterói, RJ \\ ${ }^{2}$ Faculdade de Medicina Veterinária - UFF - Niterói, RJ \\ ${ }^{3}$ Centro Universitário Serra dos Órgãos - Teresópolis, RJ \\ ${ }^{4}$ Universidade do Grande Rio - Rio de Janeiro, RJ \\ ${ }^{5}$ Centro de Primatologia do Rio de Janeiro - FEEMA - Guapimirim, RJ
}

\begin{abstract}
RESUMO
Descreveu-se o sistema reprodutor feminino em três espécies de mico-leão Leontopithecus (Lesson 1840), cativos: $L$. rosalia, $L$. chrysopygus e $L$. chrysomelas. A vulva está delimitada pelos lábios vulvares menores e com clitóris conspícuo. A superfície do períneo urogenital apresenta elevações papilares mais concentradas nos lábios vulvares, conferindo-lhe aspecto rugoso. $\mathrm{O}$ vestíbulo vaginal constitui um tubo muscular de parede espessa que se estende da rima da vulva até o óstio da vagina. A vagina é um tubo muscular alongado e achatado dorsoventralmente, que comunica o vestíbulo vaginal ao colo uterino. $\mathrm{O}$ útero piriforme está localizado na porção caudal da cavidade abdominal. Craniolateralmente abrem-se tubas uterinas convolutas e ovários grosseiramente fusiformes de superfície lisa.
\end{abstract}

Palavras-chave: mico-leão, Leontopithecus, aparelho genital, morfologia

\begin{abstract}
It is described the female genital tract of three species of lion tamarin: Leontopithecus rosalia, L. chrysopygus, and L. chrysomelas. Fifteen animals were selected from the Museum of the Center of Primatology of Rio de Janeiro - CPRJ/FEEMA. The vulva is delimited by the labia and has a conspicuous clitoris. The surface of the urogenital perineum has papillary elevations more concentrated in the labia, which results in a rough aspect. The vestibule is a thick-walled muscular tube, extending from the pudendal cleft to the vaginal orifice. The vagina is an elongated and flat muscular tube, which communicates dorsoventrally the vestibulum and the cervix of uterus. The pyriform uterus is located in the caudal portion of the abdominal cavity. Craniolaterally, the convolute uterine tubes open, enveloping the ovaries, which are roughly fusiform with a smooth surface.
\end{abstract}

Keywords: lion tamarin, Leontopithecus, genital tract, morphology

\section{INTRODUÇÃO}

A fauna primatológica mundial é rica, e o Brasil destaca-se, pois é o que possui maior diversidade de espécies de símios do mundo (Coimbra-Filho, 1983; Mittermeier et al., 1994). Na família Callitrichidae, os micos-leões (Leontopithecus) são as espécies de maior porte e habitam os remanescentes da Mata Atlântica brasileira. O Leontopithecus rosalia, presente no estado do
Rio de Janeiro, e o Leontopithecus chrysomelas, no sudeste da Bahia, apresentam-se em perigo de extinção. Já o Leontopithecus chrysopygus, original do estado de São Paulo, e o Leontopithecus caissara, endêmico no extremo norte do Paraná e no estado de São Paulo, encontram-se criticamente ameaçados, segundo o livro da Fauna Brasileira Ameaçada de Extinção (Machado et al., 2005).

Recebido em 4 de agosto de 2008 
Atualmente, alguns primatas neotropicais, como Callithrix sp., Saimiri sp., Aotus sp., vêm sendo muito estudados em pesquisas biomédicas, porém pouco se conhece sobre a biologia e a morfologia reprodutiva das formas do gênero Leontopithecus, para um melhor entendimento desses símios (Pinder e Pissinatti, 1991; Pissinatti et al., 1992, 1993; Burity et al., 1997).

Pela falta de conhecimento e escassez de literatura sobre a morfologia e a anatomia de Leontopithecus, decidiu-se estudar a morfologia do sistema reprodutor feminino de três espécies: L. rosalia, L. chrysopygus e L. chrysomelas, uma vez que essas espécies são consideradas como em perigo de extinção pela IUCN (The World Conservation of Union).

\section{MATERIAL E MÉTODOS}

Os animais selecionados para esse estudo morreram de diferentes causas em cativeiro, as quais não afetaram a morfologia do aparelho reprodutivo. Todos estavam preservados em formol tamponado a $10 \%$ e $\mathrm{pH} 7,2$ e fazem parte do acervo do Museu de Primatologia do Centro de Primatologia do Rio de Janeiro CPRJ/FEEMA.

Foram selecionadas 15 peças (Tab. 1), cinco fêmeas adultas de cada espécie. $O$ sistema reprodutor feminino de L. rosalia foi descrito e comparado com o das outras duas espécies estudadas.

Durante a execução do estudo, foi necessária a abertura da cavidade abdominal com incisão na altura da linha mediana ventral, rebatendo-se a pele e a musculatura da parede abdominal. Posteriormente, a cavidade pélvica foi aberta na região da sínfise púbica e realizada a remoção do aparelho reprodutor feminino para ser analisado e descrito individualmente. Para a observação dos detalhes e dissecação das peças, usou-se lupa esterioscópica marca Ziess nos aumentos de $2 \mathrm{x}$, $4 x$ e $8 x$.

Tabela 1. Relação de exemplares Leontopithecus com identificação e descrição dos aspectos biológicos das amostras obtidas do Museu de Primatologia do CPRJ/FEEMA

\begin{tabular}{ccccccc}
\hline Animal & Espécie & $\begin{array}{c}\text { Data de } \\
\text { nascimento }\end{array}$ & $\begin{array}{c}\text { Data de } \\
\text { morte }\end{array}$ & $\begin{array}{c}\text { Idade } \\
(\text { anos })\end{array}$ & $\begin{array}{c}\text { Peso } \\
(\mathrm{g})\end{array}$ & $\begin{array}{c}\text { Comprimento } \\
(\mathrm{mm})\end{array}$ \\
\hline 090 & L. rosalia & $16 / 08 / 74$ & $06 / 10 / 77$ & 3 & 492 & 615 \\
099 & L. rosalia & $27 / 03 / 74$ & $27 / 10 / 77$ & 3 & 483 & 586 \\
499 & L. rosalia & $23 / 06 / 80$ & $25 / 01 / 85$ & 4 & 396 & 590 \\
766 & L. rosalia & $01 / 10 / 88$ & $30 / 03 / 90$ & 1 & 418 & 530 \\
874 & L. rosalia & $15 / 09 / 89$ & $20 / 09 / 91$ & 2 & 533 & 560 \\
730 & L.chrysomelas & $16 / 10 / 86$ & $27 / 12 / 89$ & 3 & 672 & 575 \\
809 & L.chrysomelas & $28 / 11 / 82$ & $10 / 11 / 90$ & 8 & 528 & 572 \\
882 & L.chrysomelas & $06 / 10 / 89$ & $05 / 11 / 91$ & 2 & 487 & 311 \\
1395 & L.chrysomelas & $17 / 01 / 95$ & $10 / 07 / 99$ & 4 & 450 & 555 \\
1426 & L.chrysomelas & $03 / 12 / 90$ & $27 / 12 / 99$ & 9 & 360 & 595 \\
819 & L.chrysopygus & $02 / 09 / 87$ & $03 / 11 / 91$ & 4 & 553 & 590 \\
913 & L.chrysopygus & $10 / 10 / 90$ & $27 / 06 / 93$ & 2 & 405 & 600 \\
1015 & L.chrysopygus & $07 / 10 / 90$ & $27 / 06 / 93$ & 2 & 440 & 608 \\
1482 & L.chrysopygus & $14 / 01 / 96$ & $13 / 03 / 02$ & 6 & 570 & 665 \\
1551 & L.chrysopygus & $15 / 11 / 97$ & $25 / 09 / 03$ & 5 & 685 & 670 \\
\hline
\end{tabular}

\section{RESULTADOS E DISCUSSÃO}

A genitália externa de Leontopithecus está localizada no períneo urogenital, sendo rósea em L. rosalia e esbranquiçada em $L$. chrysomelas e L. chrysopygus (Fig. 1). A vulva é delimitada pelos lábios vulvares, ao contrário do que foi descrito por Hill (1957) e Hershkovitz, (1977), que afirmaram existir lábios maiores.

O clitóris é conspícuo com corpo curto e bulboso, embora nos calitriquídeos, em geral, 
não seja facilmente visível, pois este é encoberto pelos lábios vulvares (Hill, 1958). Já em cebideos, muitas vezes a hipertrofia do clitóris dificulta a sexagem de certos táxons (Pocok, 1920; Wislock, 1936; Hill, 1957; Dixson, 1998).

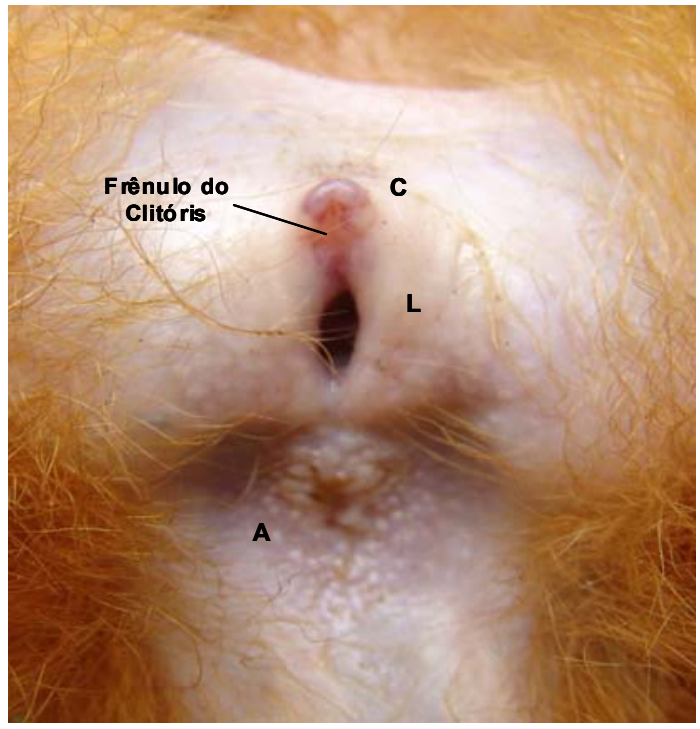

Figura 1. Genitália externa de Leontopithecus rosalia, fêmea - C: clitóris; L: lábio; A: ânus.

A superfície do períneo urogenital apresenta elevações papilares que são mais concentradas nos lábios, o que confere à vulva um aspecto rugoso. Em Callithrix jacchus e em $C$. penicillata, a região glandular é mais extensa (Pocok, 1920; Wislock, 1930).

O vestíbulo consiste em um tubo muscular de parede mais espessa, que se estende da rima da vulva até o óstio da vagina. Em sua luz, são observadas cristas longitudinais bem definidas. No entanto, os autores que descreveram o aparelho genital feminino nos calitriquídeos consideraram esse segmento do trato genital como uma porção da vagina (Hill, 1957; Cui e Matthews, 1994).

A vagina apresenta-se como um tubo muscular alongado e achatado dorsoventralmente e faz comunicação com o vestíbulo, caudalmente, e com o colo do útero, cranialmente. A vagina é longa em Leontopithecus, assim como em outros calitriquídeos, em razão direta de o útero estar posicionado na cavidade abdominal, diferentemente do que foi observado em outros símios, como chimpanzé e gorila, nos quais o útero localiza-se na cavidade pelvina (Dixson,
1998). Na luz vaginal, no sentido transversal, nota-se um pregueamento em toda sua extensão. No terço final da vagina, observa-se a uretra como um tubo muscular percorrendo a parede ventral, abrindo-se como um óstio no lúmen vaginal.

O útero (Fig. 2) apresenta-se piriforme e está localizado na porção caudal da cavidade abdominal. Craniolateralmente abrem-se as tubas uterinas, uma de cada lado, e caudalmente o útero se continua com a vagina. $\mathrm{O}$ fundo do útero está cranialmente às aberturas das tubas uterinas. A tuba uterina é um tubo muscular e convoluto. Abre-se na cavidade peritonial através do óstio abdominal da tuba uterina e apresenta processos fibrosos, as fímbrias. Nos gêneros Callithrix, Saimiri e Lagothrix, as tubas apresentaram-se retilíneas (Wislock, 1932; Hill, 1953).

Os ovários (Fig. 2) apresentam-se grosseiramente fusiformes e de superfície lisa, com faces dorsal, lateral e ventral, e estão posicionados cranialmente ao útero, lateralmente ao cólon sigmóide e dorsalmente às tubas uterinas. $\mathrm{Na}$ maioria dos Platyrrhini, os ovários apresentam-se na forma ovóide, exceto em Callithrix sp, pois é ligeiramente arredondado (Hill, 1953).

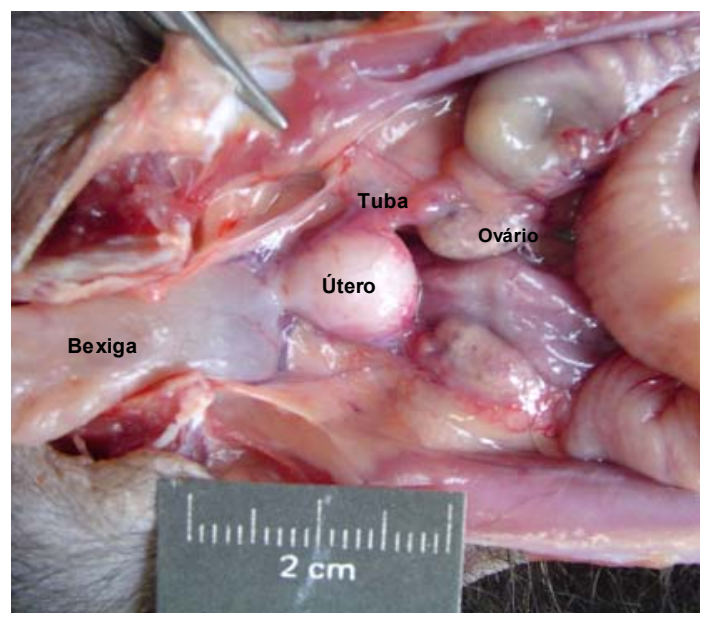

Figura 2. Cavidade abdominal de Leontopithecus chrysomelas, fêmea.

Em resumo, em Leontopithecus a vulva é delimitada pelos lábios vulvares, o vestíbulo vaginal está presente, a vagina é alongada, o útero localiza-se na cavidade abdominal, as tubas uterinas são convolutas e os ovários são fusiformes. 


\section{AGRADECIMENTOS}

À coordenação de curso de pós-graduação da Faculdade de Medicina Veterinária - UFF. Ao Centro de Primatologia do Rio de Janeiro CPRJ/FEEMA. A Fundação Carlos Chagas Filho de Amparo à Pesquisa do Estado do Rio de Janeiro - FAPERJ (Proc. No E-26/171.185/2004). Ao Greater Los Angeles Zoo Association (GLAZA) e ao IBAMA, pela constante cooperação no programa de reprodução de primatas do neotrópico.

\section{REFERÊNCIAS BIBLIOGRÁFICAS}

BURITY, C.H.F.; MANDARIM DE LACERDA, C.A.; PISSINATTI, A. Craniometric sexual dimorphism in Leontopithecus Lesson, 1840 (Callithrichidae, Primates). Primates, v.38, p.185-96, 1997.

COIMBRA-FILHO, A.F. Situação atual dos calitriquídeos que ocorrem no Brasil. Callitrichidae Primates. In: A primatologia no Brasil. Belo Horizonte: Sociedade Brasileira de Primatologia, 1983. p.15-33.

CUI, K.; MATTHEWS, C.D. Anatomy of adult female cammon marmoset (Callitrhix jacchus) reprodutive system. J. Anat., v.185, p.481-486, 1994.

DIXSON, A.F. (Ed). Primates sexuality: comparative studies of the prosimians, monkeys, apes and humans beings. New York: Oxford University, 1998. 546p.

HERSHKOVITZ, P. (Ed). Living new world monkeys (Platyrrhini). Chicago: Chicago University, 1977. v.1, $1117 \mathrm{p}$.

HILL, W.C.O. Observations on the genitalia of the woolly monkey (Lagothrix). Proc. Zool. Soc. London, v.122, p.973-984, 1953.
HILL, W.C.O. (Ed) Primates comparative anatomy and taxonomy. Edinburgh: Edinburgh University, 1957. 354p.

HILL, W.C.O. (Ed). Primatologia handbook of primatology. New York: S. Kanger, 1958. 704p.

MACHADO, A.B.M.; MARTINS, C.S.; DRUMMOND, G.M. Lista da fauna brasileira ameaçada de extinção. Belo Horizonte: Fundação Biodiversitas, 2005. 157p.

MITTERMEIER, R.A.; KONSTANT, W.R.; MAST, R.B. Use of neotropical and malgasy primates species in biomedical research. Am. J. Primates, v.34, p.73-80, 1994.

PINDER, L.; PISSINATTI, A. Malformações congênitas em Leontopithecus rosalia (Linnaeus, 1766). Callitrichidae - Primates. In: A primatologia no Brasil 3. Belo Horizonte: Sociedade Brasileira de Primatologia, 1991. p.191-195.

PISSINATTI, A.; SILVA, E.C.; COIMBRA-FILHO, A.F. et al. Sexual dimorphism of the pelvis in Leontopithecus (Lesson, 1840). Folia Primatol., v.58, p.204-209, 1993

PISSINATTI, A.; CRUZ, J.B.; NASCIMENTO, M.D. et al. Spontaneous gallstones in marmosets and tamarins (Callitrichidae-Primates). Folia Primatol., v.59, p.44-50, 1992.

POCOK, R.I. On the external characters of the South American Monkey. Proc. Zool. Soc. London, v.3, p.91-113, 1920.

WISLOCKI, G.B. A study of scent glands in the marmosets, especially Oedipomidas geoffroyi. J. Mammal., v.11, p.475-483, 1930.

WISLOCKI, G.B. On the female reproductive tract of the gorilla, with comparison of that of others primates. Contr. Embryol. Carneg. Inst., v.23, p.163-204, 1932.

WISLOCKI, G.B. The external genitalia of the simian primates. Hum. Biol., v.8, p.309-347, 1936. 